Revista lus et Praxis, Año 16, N² 2, 2010, pp. 331 - 352

ISSN 0717 - 2877

Universidad de Talca - Facultad de Ciencias Jurídicas y Sociales

"Licencias obligatorias por razones de salud pública en Chile.

Un análisis comparativo con el acuerdo sobre los ADPIC"

Alberto Cerda Silva

\title{
LICENCIAS OBLIGATORIAS POR RAZONES DE SALUD PÚBLICA EN CHILE. UN ANÁLISIS COMPARATIVO CON EL ACUERDO SOBRE LOS ADPIC*-**
}

\author{
COMPUlSORY LICENSES FOR REASONS OF PUBliC HEALTH IN CHILE. \\ A COMPARATIVE ANALYSIS WITH THE TRIPS AGREEMENT
}

Alberto Cerda Silva***

\section{RESUMEN}

En la implementación de sus múltiples acuerdos internacionales en materia de propiedad intelectual, Chile ha modificado su Ley de Propiedad Industrial, la cual reglamenta el sistema de patentes, reconociendo la patentabilidad de productos farmacéuticos (1991) y estableciendo un mecanismo para el otorgamiento de licencias obligatorias fundadas en razones de salud pública (2005). Este artículo

describe y analiza la normativa sobre licencias obligatorias sobre patentes farmacéuticas adoptadas en Chile en orden a establecer si ellas satisfacen el estándar adoptado por la Organización Mundial de Comercio (OMC) en el Acuerdo sobre los Aspectos de los Derechos de Propiedad Intelectual relacionados con el Comercio (ADPIC) y saca partido de las flexibilidades provistas por los instrumentos internacionales, en especial aquellos que resultaron de la Declaración de Doha.

\section{ABSTRACT}

In implementing its various international agreements on intellectual property, Chile has amended its industrial property act, which regulates the patent system, by recognizing the patentability of pharmaceutical products (1991) and establishing a scheme for granting compulsory licenses based on reasons of public health (2005). This article describes and analyses the rules adopted about compulsory licenses on pharmaceutical patents in order to establish whether it meets the standard promoted by the World Trade Organization in the Agreement on Trade Related Aspects of Intellectual Property Rights (TRIPS) and takes advantages of flexibilities provided by international agreements, especially those resulting of the Doha Declaration.

$$
\text { PALABRAS Clave }
$$

ADPIC, Patentes Farmacéuticas, Licencias Obligatorias

KEY WORDS

TRIPS, Pharmaceutical Patents, Compulsory Licenses

\footnotetext{
* Trabajo recibido el 16 de julio y aprobado el 10 de agosto de 2010.

** El autor desea agradecer a la Fulbright Commission y la Comisión Nacional de Ciencia y Tecnología, por su apoyo para la realización del Programa de LL.M. in International Legal Studies en Georgetown University Law Center, 2009-2010, en el marco del cual tuvo la ocasión de escribir el paper del mismo nombre que, con ligeras modificaciones, corresponde al presente artículo.

*** Licenciado en Ciencias Jurídicas y Sociales, Universidad de Chile; Magíster en Derecho Público, Universidad de Chile; LL.M. in International Legal Studies, Georgetown University; Profesor en Derecho Informático, Facultad de Derecho de la Universidad de Chile. Correo electrónico: acerda@uchile.cl.
} 


\section{ANTECEDENTES}

Hasta 1991, Chile rechazaba la patentabilidad de los productos farmacéuti$\cos ^{1}$. Ese año, el país adoptó una nueva normativa sobre propiedad industrial ${ }^{2}$, la cual garantizó la patentabilidad de dichos productos ${ }^{3}$. Sin embargo, la nueva ley no estableció medidas de resguardo para hacer frente a situaciones críticas de salud pública ${ }^{4}$, particularmente la ley no adoptó normas que permitiesen el otorgamiento de licencias obligatorias sobre patentes farmacéuticas (i.e., autorización de la autoridad pública para explotar una patente sin autorización de su titular, pero con el pago de una remuneración) $)^{5}$, aunque si para casos de abuso monopólico ${ }^{6}$.

${ }^{1}$ El artículo $5^{\circ}$ del Decreto Ley $N^{\circ}$ 958, de 1931, sobre Propiedad Industrial, publicado en el Diario Oficial el 27 de julio de 1931, excluía expresamente de patentabilidad los medicamentos de toda especie y las preparaciones farmacéuticas medicinales.

${ }^{2}$ Ley $N^{\circ} 19.039$, establece normas aplicables a los privilegios industriales y protección de los derechos de propiedad industrial, publicada en el Diario Oficial el 25 de enero de 1991.

${ }^{3}$ Selt, Susan, Private Power, Public Law: The Globalization of Intellectual Property Rights, Cambridge Studies in International Relations, Cambridge, 2003, pp. 1-2, quien describe la adopción de patentes farmacéuticas en Chile como un logro de la asociación de empresas farmacéuticas estadounidense.

${ }^{4}$ Con todo, la actual ley aún excluye la patentabilidad de los métodos de tratamiento quirúrgico o terapéutico del cuerpo humano o animal, así como los métodos de diagnóstico aplicados al cuerpo humano o animal, salvo los productos destinados a poner en práctica uno de estos métodos. Vid. artículo 37 d) Ley № 19.039.

${ }^{5}$ Durante la discusión legislativa del proyecto de Ley № 19.039, la Cámara de Diputados aprobó un artículo que declaraba de utilidad pública y autorizaba la expropiación mediante decreto de las patentes de invención que afectaran el derecho a vivir en un medio ambiente libre de contaminación y el derecho a la protección de la salud, establecidos en el artículo 19 de la Constitución Política. Expropiada la patente, su titular sería el Fisco, quien podría otorgar licencias gratuitas a todo el que lo solicitara. INFORME DE LA COMISIÓN DE ECONOMÍA, FOMENTO y DeSARROLlo recaído en el proyecto de ley sobre propiedad industrial, 18 de Julio de 1990, p. 42. En: http://sil.congreso.cl/cgi-bin/sil_abredocumentos. pl?2,1273 [visitado el 21/07/2010]. La mencionada propuesta fue rechazada por el Senado por ser muy genérica. INFORME DE LA COMISIÓN DE ECONOMÍA, Fomento Y RECONSTRUCCIÓN recaído en el proyecto de Ley de la H. Cámara de Diputados sobre Propiedad Industrial, 12 de septiembre de 1990, p. 20. En: http:// sil.congreso.cl/cgi-bin/sil_abredocumentos.pl?2,1758 [visitado el 21/07/2010].

Finalmente, la Comisión Mixta estimó que de presentarse una aguda escasez de medicamentos, ésta debía resolverse mediante compras públicas y, por otra parte, la Constitución ya contempla la facultad de expropiar por causa de utilidad pública o de interés nacional, calificada por el legislador, en virtud de ley general o especial. En otros términos, la expropiación debe tener lugar a través de ley y no de un simple decreto. Vid. Informe de la Comisión Mixta Relativo al Proyecto de Ley sobre Propiedad INDUSTRIAL, 14 de noviembre de 1990, pp. 7-9. En: http://sil.congreso.cl/cgi-bin/sil_abredocumentos. pl?2,3094 [visitado el 21/07/2010].

${ }^{6}$ Artículo 51 Ley № 19.039. En caso de abuso monopólico, la ley facultaba el otorgamiento de licencia obligatoria por la autoridad encargada de velar por la libre competencia: la Comisión Resolutiva, organismo encargado de supervigilar la adecuada aplicación de las normas del Decreto Ley № 211, de 1973, que fijó normas para la defensa de la libre competencia. Ésta debía, cuando menos, calificar 
Posteriormente, con la aspiración de lograr apertura en los mercados internacionales, Chile suscribió y adhirió a diversos acuerdos internacionales, varios de los cuales incluyen temas de propiedad intelectual. De entre ellos, en materia de patentes, los más relevantes son el Convenio de Paris ${ }^{7}$ y el Acuerdo sobre los Aspectos de los Derechos de Propiedad Intelectual Relacionados con el Comercio $(\mathrm{ADPIC})^{8}$. Dado que ninguno de dichos acuerdos es auto-ejecutable ${ }^{9}$,

la existencia de una situación de abuso monopólico y, en su caso, establecer las condiciones en que el licenciatario debería explotar la patente, su duración, y la compensación que debía pagarse al titular de la patente. La ley también daba indicaciones para la fórmula de cómputo de la compensación. La regulación de las licencias no voluntarias era bastante escueta en la ley.

${ }^{7}$ Chile adhirió al Convenio de París el 13 de marzo de 1991 y éste entró en vigencia el 14 de junio de 1991. El convenio fue promulgado mediante Decreto $N^{\circ}$ 425, que promulga el Convenio de París para la Protección de la Propiedad Industrial, adoptado en París el 20 de marzo de 1883, publicado en el Diario Oficial de 30 de septiembre de 1991.

${ }^{8}$ Chile adhirió al Acuerdo de Marrakech el 28 de diciembre de 1994. El Acuerdo fue promulgado mediante Decreto $\mathrm{N}^{\circ} 16$, que promulga el Acuerdo de Marrakech, por el que se establece la Organización Mundial de Comercio, y los Acuerdos Anexos que se indican, publicado en el Diario Oficial de 17 de mayo de 1995.

${ }^{9}$ Aun no siendo objeto principal de este artículo, es conveniente referirse siquiera brevemente a si el Acuerdo sobre los ADPIC, y particularmente su artículo 31, son auto-ejecutables o no. Un tratado internacional es auto-ejecutable cuando sus disposiciones son incorporadas en la legislación interna permitiendo exigir directamente su cumplimiento ante los tribunales, aun cuando no exista una ley que le implemente. El carácter auto-ejecutable o no de un tratado depende esencialmente de cada sistema jurídico, en especial de su marco constitucional. Precisamente, porque los países disponen de diferentes regímenes normativos, el Acuerdo sobre los ADPIC reconoce la libertad de las partes para determinar el método de implementación apropiado en su propio sistema y prácticas legales, esto es, si confieren al tratado o a sus disposiciones carácter auto-ejecutable o no. Vid., UNCTAD-ICTSD, Resource Book on TRIPS and Development, Cambridge University Press, Nueva York, 2005, pp. 17 et seq.; CORREA, Carlos, "The TRIPS Agreement and Developing Countries", en MaCrony y otros (Edits.), The World Trade Organization: Legal, Economic and Political Analysis, Volumen 2, Springer, New York, 2005, pp. 434-435, y Gervals, Daniel, The TRIPS Agreement: Drafting, History and Analysis, $3^{a}$ Edición, Thomson Reuters, Londres, 2008, pp. 164-165. Así, por ejemplo, en el caso de Estados Unidos, la doctrina está conteste en privar de carácter auto-ejecutable al Acuerdo sobre los ADPIC. Vid., Brennan, David J., Retransmission and US Compliance with TRIPS, Kluwer Law International, La Haya, Holanda, 2003, pp. 314-315; HAsson, Adam Isaac, "Domestic Implementation of International Obligations: the Quest for World Patent Law Harmonization", 25 Boston College International and Comparative Law Review 373, 2002, y WEGNER, Harold C., "Injunctive Relief: A Charming Betsy Boomerang", en Northwestern Journal of Technology and Intellectual Property Volumen 4, Número 2, 2006, pp. 166-167. Sin embargo, aun cuando algunos países confieran carácter auto-ejecutable a los tratados internacionales, es posible que ciertas disposiciones del Acuerdo sobre los ADPIC requiera la adopción de normas de implementación. WATAL, Jayashree, Intellectual Property Rights in the WTO and Developing Countries, Kluwer Law International, La Haya, Holanda, 2001, p. 49; MONCAYO vON HASE, Andrés, "The Application and Interpretation of the Agreement on Trade-Related Aspect of Intellectual Property Rights", en CORREA, Carlos; Yusuf, Abdulqawi (Edits.), Intellectual Property and International Trade: the TRIPs Agreement, 2a Edición, Wolters Kluwer, Holanda, 2008, pp. 105 y et seq. Éste parece ser el caso, según nuestro entender, del artículo 31 de los ADPIC. En contra. vid. PIRES DE Carvallo, Nuno, The TRIPS Regime on Patent Rights, $2^{a}$ Edición, Kluwer Law International, Holanda, 
su adhesión ha requerido adoptar normas de implementación en el derecho interno. Dicho proceso se ha llevado a cabo progresivamente, empezando en 1999, cuando el Gobierno presentó al Congreso un proyecto de ley que implementaba el Convenio de París y el Acuerdo sobre los ADPIC, modificando la Ley de Propiedad Industrial vigente.

Durante los años en que estuvo en discusión el proyecto de ley en el Congreso, la Organización Mundial de Comercio (OMC) adoptó la Declaración de Doha ${ }^{10}$ y la Decisión sobre su aplicación ${ }^{11}$, Chile avanzó en la negociación de acuerdos comerciales con Estados Unidos y la Unión Europea ${ }^{12}$, el mundo conoció de los estragos del HIV y el Ébola en los países no desarrollados, y Estados Unidos experimentó el peligro de ataques bacteriológicos con Ántrax tras el 11 de Septiembre. Cada uno de dichos hechos tuvo incidencia en el debate legislativo ${ }^{13}$.

2005, pp. 59-60, quien, en su afán por dejar a resguardo los derechos del titular de la patente, obvia que, aun no siendo auto-ejecutable una disposición de un tratado en el derecho interno de un país, este es responsable en el foro internacional por su eventual infracción.

En el caso de Chile, aun cuando la Constitución admite la ejecución directa de los tratados internacionales ratificados y que se encuentren vigentes, ella se limita a aquellos que fijan derechos esenciales que emanan de la naturaleza humana, carácter que difícilmente puede atribuirse al Acuerdo sobre los ADPIC y al Convenio de París. De hecho, como ha quedado dicho, no existe disposición alguna en el Acuerdo sobre los ADPIC que establezca que este es auto-ejecutable. Por el contrario, por su propia naturaleza y por el alcance de varias de sus disposiciones, el Acuerdo requiere la adopción de normas de derecho interno, lo cual obsta a que sea auto-ejecutable (Vid., especialmente los artículos 1.1, 8.1, y 63.2). Pero, aun en el caso de que el Acuerdo sobre los ADPIC fuese auto-ejecutable, éste no sería el caso de su artículo 31, que reglamenta las licencias obligatorias, ya que, por su propio objetivo y por los términos del mismo, supone cierta implementación a nivel de derecho interno.

${ }^{10}$ Declaración relativa al Acuerdo sobre los ADPIC y la salud pública, adoptada en Doha el 14 de noviembre de 2001. WT/MIN(01)/DEC/2.

${ }^{11}$ Decisión del Consejo General de 30 de agosto de 2003, sobre Aplicación del párrafo 6 de la Declaración de Doha relativa al Acuerdo sobre los ADPIC y la salud pública. WT/L/540 y Corr.1.

${ }^{12}$ Acuerdo de Asociación Política, Económica y de Cooperación entre Chile y la Unión Europea, suscrito en Bruselas el 18 de noviembre de 2002, mediante el cual Chile se obligó a adherir e implementar diversos acuerdos internacionales en materia de propiedad intelectual. De entre ellos, cabe destacar el Tratado de Cooperación en Materia de Patentes (Washington 1970, enmendado en 1979 y modificado en 1984); y el Arreglo de Estrasburgo relativo a la Clasificación Internacional de Patentes (Estrasburgo 1971, modificado en 1979). Vid., artículo 170 del Acuerdo. Por su parte, el Tratado de Libre Comercio entre Chile y Estados Unidos, suscrito el 6 de junio de 2003, mediante el cual Chile convino en adoptar determinadas normas de protección de tales derechos más allá de las previstas en los acuerdos multilaterales.

${ }^{13}$ En la discusión legislativa se aludió continuamente a los compromisos internacionales asumidos por Chile, e igualmente al uso de licencias obligatorias por Brasil y Sudáfrica, y al peligro de ataques bacteriológicos en Estados Unidos. Así, por ejemplo, en la Cámara de Diputados, Sesión 32, Legislatura 345, 5 de marzo de 2002, pp. 32-34. En: http://sil.congreso.cl/cgi-bin/sil_abredocumentos.pl?5,4080 [visitado el 21/07/2010]. 
En tal contexto, pese a que la iniciativa legal inicialmente no contemplaba novedad alguna en relación a las licencias no voluntarias, durante la tramitación legislativa se decidió establecer un nuevo régimen de licencias obligatorias, para garantizar su otorgamiento, entre otros casos, por razones de "salud pública, seguridad nacional, uso público no comercial, o de emergencia nacional u otras de extrema urgencia".$^{14}$ ¿Cuál es el alcance y el tratamiento que la legislación chilena hace de las licencias obligatorias para fines farmacéuticos? ¿Está dicha regulación en armonía con el Acuerdo sobre los ADPIC? ¿Aprovecha Chile de las flexibilidades explicitadas en la Declaración de Doha y demás instrumentos subsecuentes a ella?

\section{El PROCESO LEGISLATIVO DE LAS LICENCIAS ObLIGATORIAS}

A finales de 1999, días antes de vencer el plazo inicialmente previsto en el Acuerdo sobre los ADPIC para la implementación en derecho interno de sus disposiciones, el Gobierno de Chile presentó un proyecto de ley al Congreso, mediante el cual modificaba significativamente la Ley de Propiedad Industrial ${ }^{15}$, a efectos de cumplir con lo dispuesto en tal acuerdo internacional y en el Convenio de París ${ }^{16}$. En materia de licencias obligatorias, el proyecto no introducía novedades respecto de la legislación anterior ${ }^{17}$, lo cual fue criticado por las asociaciones de propiedad intelectual ${ }^{18}$ y farmacéuticas nacionales ${ }^{19}$.

Las críticas antes mencionadas llevaron a la introducción de diversas indicaciones específicas sobre la materia en la Cámara de Diputados, precisamente en los días previos a la Declaración de Doha ${ }^{20}$. La inclusión de disposiciones

\footnotetext{
${ }^{14}$ Artículo 51 número 2) Ley № 19.039, modificada por la Ley № 19.996.

${ }^{15}$ De acuerdo al a la Nota No 727, de 28 de diciembre de 1994, de la Misión de Chile ante los Organismos Internacionales en Ginebra, se informó a la Secretaría del Acuerdo General sobre Aranceles Aduaneros y Comercio el deseo del Gobierno de Chile de disponer de los plazos máximos posibles para la plena aplicación de las obligaciones del Acuerdo para países en desarrollo.

${ }^{16}$ Mensaje de S.E. el Presidente de la República, con el que inicia un proyecto de ley que modifica la Ley № 19.039, 4 de octubre de 1999, pp. 4-5. En: http://sil.congreso.cl/cgi-bin/sil_abredocumentos.pl?1,1200 [visitado el 21/07/2010].

${ }^{17}$ Salvo el caso mencionado precedentemente de licencias obligatorias otorgadas en caso de prácticas monopólicas. Vid, nota al pie supra 6.

${ }^{18}$ La Asociación Chilena de la Propiedad Industrial (ACHIPI). Vid., INFORME dE LA COMISIÓN DE ECONOMía, Fomento y DesARROLlo (de la Cámara de Diputados) recaído en el proyecto de ley que modifica la Ley 19.039, que establece normas aplicables a los privilegios industriales y protección de los derechos de propiedad industrial, 8 de agosto de 2001, p. 18. En: http://sil.congreso.cl/cgi-bin/sil_abredocumentos. pl?2,2867 [visitado el 21/07/2010].

${ }^{19}$ La Asociación Industrial de Laboratorios Farmacéuticos (ASILFA). Vid., INFORME DE LA COMISIÓN DE ECONOMíA, cit. nota. n. 18, p. 26.

${ }^{20}$ InFORMe de LA Comisión de ECONOMía, cit. nota. n. 18, pp. 94-96.
} 
sobre licencias obligatorias por razones de "salud pública" generó diversas reacciones en el medio nacional. La iniciativa fue calificada de innecesaria por la asociación de farmacéuticas internacionale ${ }^{21}$, mientras que recibió el respaldo de la industria local y otras asociaciones ${ }^{22}$. Por su parte, el Gobierno reiteró que la iniciativa se encontraba en "armonía" con los Acuerdos sobre los ADPIC ${ }^{23}$; de hecho, el texto de la propuesta seguía muy de cerca la redacción del mencionado Acuerdo.

A finales de 2001, existían aún dudas e incertidumbres en el Congreso en cuanto a la consistencia de la iniciativa de ley con los compromisos internacionales de Chile. Éstas no eran sino reflejo de la falta de certeza y de la presión recibida por los países en desarrollo para adoptar medidas de protección a la salud pública, particularmente licencias obligatorias, en desmedro de los derechos de propiedad intelectual ${ }^{24}$. En noviembre de 2001, tal situación condujo a la adopción de la Declaración de Doha, la cual clarificó que el Acuerdo sobre los ADPIC no impide a los Miembros adoptar medidas para proteger la salud pública y reafirmó el derecho de éstos para utilizar, al máximo, las disposiciones del Acuerdo que prevén flexibilidad a este efecto ${ }^{25}$.

En 2002, al reanudarse la discusión legislativa existía certidumbre de que las licencias obligatorias pueden ser adoptadas por causas de salud pública y el Congreso se concentró en la redacción de las disposiciones específicas contempladas en el proyecto sobre la materia ${ }^{26}$. Finalmente, en 2005, tras ser

\footnotetext{
${ }^{21}$ La Cámara de la Industria Farmacéutica de Chile A.G., que agrupa a 20 industrias farmacéuticas internacionales, incluidas Abbott, Bayer, Bristol-Myers Squibb, Merck, Novartis, Pfizer y Roche, entre otras. Vid., INFORME DE LA COMIIIÓN DE HACIENDA (de la Cámara de Diputados) recaído en el proyecto de ley que modifica la Ley $N^{\circ} 19.039$, que establece normas aplicables a los privilegios industriales y protección de los derechos de propiedad industrial, 9 de noviembre de 2001, p. 4. En: http://sil. congreso.cl/cgi-bin/sil_abredocumentos.pl?2,3465 [visitado el 21/07/2010].

${ }^{22}$ Informe de la Comisión de HaCIenda, cit. nota n. 21, pp. 7-9. También en Nuevo Informe de la Comisión de Economía, Fomento y Desarrollo (de la Cámara de Diputados) recaído en el proyecto de ley que modifica la Ley $N^{\circ} 19.039$, que establece normas aplicables a los privilegios industriales y protección de los derechos de propiedad industrial, 12 de enero de 2002, pp. 21 y 29. En: http://sil.congreso.cl/ cgi-bin/sil_abredocumentos.pl?2,3644 [visitado el 21/07/2010].

${ }^{23}$ Nuevo Informe de la Comisión de Economía, cit. nota n. 22, p. 7.

${ }^{24}$ Así algunos países han sufrido medidas unilaterales de "retaliación" y suspensión de ayuda. CoRREA, Carlos, "Pro-competitive Measures under TRIPS to Promote Technology Difussion in Developing Countries", en Drahos, Peter; Marne, Ruth (Edits.), Global Intellectual Property Rights. Knowledge, Access and Development, Palgrave Macmillan, Nueva York, 2002, p. 50.

${ }^{25}$ Numeral 4 de la Declaración relativa al acuerdo sobre los ADPIC y la salud pública.

${ }^{26}$ La última voz disonante en la materia fue de la Cámara de la Industria Farmacéutica, que insistió en que las licencias obligatorias constituyen una excepción al derecho de propiedad, por lo cual deben ser estrictas y restringidas; además, la mencionada asociación enfatizó que, aun cuando permitidas en ADPIC, Chile no está obligado a incluirlas. Vid., INFORME DE LA COMISIÓN DE ECONOMía
} 
aprobado por ambas cámaras del Congreso, se publicó la Ley № 19.996 que modificó la Ley de Propiedad Industrial en diversos puntos, incorporando el otorgamiento de licencias obligatorias por razones de salud pública ${ }^{27}$.

La nueva ley admite la concesión de licencias obligatorias en tres hipótesis: a) en casos de prácticas anticompetitivas del titular de la patente ${ }^{28}$; b) en casos de patentes dependientes ${ }^{29}$, y c) por razones de salud pública, seguridad nacional, uso público no comercial, o de emergencia nacional u otras de extrema urgencia ${ }^{30}$.

\section{LICENCIAS OBLIGATORIAS FUNDADAS EN RAZONES DE SALUD PÚBLICA EN CHILE}

De acuerdo a la normativa nacional, es posible que se otorgue una licencia obligatoria respecto de productos farmacéuticos fundada en prácticas anticompetitivas o en patentes dependientes; no obstante, en los párrafos que siguen nuestro análisis se limitará a aquellas licencias otorgadas por razones de salud pública ${ }^{31}$.

Razones de salud pública. Lo primero que salta a la vista de comparar la legislación chilena con el Acuerdo sobre los ADPIC es que los casos en los cuales procede el otorgamiento de una licencia obligatoria son más amplios que los previstos expresamente en los Acuerdos. La ley autoriza a otorgar la licencia por razones de "salud pública, seguridad nacional, uso público no comercial, o de emergencia nacional u otras de extrema urgencia".

Salud pública y seguridad nacional no son conceptos incluidos expresamente en el Acuerdo sobre los ADPIC; sin embargo, tanto del tenor literal del artículo

(del Senado) recaído en el proyecto de ley que modifica la Ley $N^{\circ} 19.039$, que establece normas aplicables a los privilegios industriales y protección de los derechos de propiedad industrial, 27 de agosto de 2002, pp. 8-11. Disponible en http://sil.congreso.cl/cgi-bin/sil_abredocumentos.pl?2,4910 [visitado el 21/07/2010].

${ }^{27}$ Ley № 19.996 que modifica la Ley № 19.039 sobre Propiedad Industrial, publicada en el Diario Oficial el 11 de marzo de 2005.

${ }^{28}$ Artículo 51 número 1) Ley № 19.039, en relación con artículo 31 k) ADPIC.

${ }^{29}$ Artículo 51 número 3) Ley № 19.039, en relación con el artículo 31 I) ADPIC.

${ }^{30}$ Artículo 51 número 2) Ley № 19.039.

${ }^{31}$ Durante la discusión legislativa se desechó una indicación destinada a limitar los casos en que procedían otorgar una licencia obligatoria a aquellos en que "el titular del derecho no se encuentra explotándolo en el territorio nacional", por sí o por un tercero. SEGUNDO INFORME DE LA COMISIÓN DE ECONOMía (del Senado) recaído en el proyecto de ley que modifica la Ley $\mathrm{N}^{\circ} 19.039$, que establece normas aplicables a los privilegios industriales y protección de los derechos de propiedad industrial, 29 de enero de 2004, p. 63. En: http://sil.congreso.cl/cgi-bin/sil_abredocumentos.pl?2,6304 [visitado el 21/07/2010]. 
31 del Acuerdo sobre los ADPIC ${ }^{32}$, como de la Declaración de Doha ${ }^{33}$, resulta claro que tales circunstancias revisten la gravedad y urgencia necesaria para justificar el otorgamiento de una licencia obligatoria.

Autoridad competente. Pese a la incertidumbre que precedió a la Declaración de Doha ${ }^{34}$, en orden a que cada país miembro tiene "el derecho de determinar lo que constituye una emergencia nacional u otras circunstancias de extrema urgencia" ${ }^{\prime 35}$, la ley chilena desde muy temprano dejó en claro que las circunstancias que justifican el otorgamiento de una licencia obligatoria son "declaradas por la autoridad competente". ${ }^{36}$

Inicialmente la autoridad competente para conceder una licencia obligatoria por razones de salud pública era el Jefe del Departamento de Propiedad Industrial. Sin embargo, a partir de 2009, dicha autoridad es el Director Nacional del recientemente creado Instituto Nacional de Propiedad Industrial (INAPI) ${ }^{37}$, quien conocerá de la solicitud conforme al procedimiento establecido para la nulidad de patentes ${ }^{38}$.

Tratativas previas. Quien solicita una licencia obligatoria debe probar haber solicitado una licencia contractual previamente a su titular, sin obtenerla en condiciones y plazo razonables ${ }^{39}$. Esta no es una exigencia en el caso de las licencias otorgadas por razones de salud pública ${ }^{40}$, lo cual es consistente con el Acuerdo sobre ADPIC, en cuanto permite eximir de dicha exigencia ante tal ca$\mathrm{So}^{41}$. Sin embargo, según el Acuerdo, en este caso el titular debe ser notificado en

\footnotetext{
${ }^{32}$ El artículo 31 de los ADPIC, titulado "otros usos sin autorización del titular de los derechos", refiere a usos distintos de los contemplados en el artículo 30 (excepciones) y que se permiten en la legislación de un Miembro, incluido el uso por el gobierno o por terceros autorizados por el gobierno. De ellos resulta que los ADPIC no han pretendido limitar los casos en los cuales procede que un país conceda licencias obligatorias.

${ }^{33}$ Número 5 b) y c) Declaración relativa al acuerdo sobre los ADPIC y la salud pública.

${ }^{34}$ Dichas dudas fueron alentadas desde el sector farmacéutico, entre otros. Аввотт, Frederick; CotтIER, Thomas; GuRRY, Francis, International Intellectual Property in an Integrated World Economy, ASPEN, Nueva York, 2007, p. 202.

${ }^{35}$ Número 5 c) Declaración relativa al acuerdo sobre los ADPIC y la salud pública.

${ }^{36}$ Artículo 51 número 2) Ley No 19.039.

${ }^{37}$ Artículo Primero Transitorio Ley № 20.254 que crea el Instituto Nacional de Propiedad Industrial, publicada en el Diario Oficial el 14 de abril de 2008.

${ }^{38}$ Artículo 51 bis B 2) Ley № 19.039.

${ }^{39}$ Artículo 51 bis A primera parte Ley № 19.039 en relación con el artículo 31 b) primera parte de ADPIC.

${ }^{40}$ Artículo 51 bis A segunda parte Ley $N^{\circ} 19.039$.

${ }^{41}$ Artículo 31 b) segunda parte ADPIC. En contra, Pires de Carvallo, para quien la omisión de las tratativas previas sólo tiene lugar en casos de "extrema" urgencia. PIRES, The TRIPS, cit. nota n. 9, pp. 325-326. Más permisivo es CORREA, "Pro-competitive", cit. nota n. 24, p. 49.
} 
cuanto sea "razonablemente posible"; dicha exigencia no aparece expresamente recogida en la ley chilena, pero se subentiende cumplida por el procedimiento que debe seguir el INAPI para el otorgamiento de la licencia ${ }^{42-43}$.

Condiciones de la licencia. Al igual que el Acuerdo sobre los ADPIC ${ }^{44}$, la ley requiere que la licencia sea otorgada en función de las circunstancias propias de ésta ${ }^{45}$. Existe acuerdo en que esta exigencia básicamente excluye una "cláusula de licenciamiento general"," 46 obligando a que su otorgamiento se verifique sobre la base de un análisis caso a caso $^{47}$.

En caso de ser otorgada, la licencia deberá fijar su duración y alcance, limitándose a aquellos fines para los cuales fue concedida, así como la remuneración concedida al titular de la patente ${ }^{48}$. La licencia será no-exclusiva, permitiéndose la explotación de la patente por su propietario, y no podrá cederse, salvo con aquella parte de la empresa titular de la patente $(\text { sic })^{49}$.

Remuneración. Uno de los elementos esenciales del otorgamiento de licencias obligatorias en el Acuerdo sobre los ADPIC es que el titular de los derechos reciba una "remuneración adecuada" según las circunstancias propias de cada caso, habida cuenta del valor económico de la autorización ${ }^{50}$. El Acuerdo no

${ }^{42}$ Artículo 51 bis B Ley № 19.039. La solicitud de licencia obligatoria debe cumplir con los requisitos de una demanda judicial, conforme las normas del Código de Procedimiento Civil, incluida la individualización de la parte demandada. Esta es presentada en el INAPI, organismo que la tramita conforme al procedimiento para nulidad de patentes establecido en la propia ley, que prevé su notificación al titular de la patente. Artículo 18 bis I Ley No 19.039.

${ }^{43}$ De acuerdo al artículo 31 b) de los ADPIC, "En el caso de uso público no comercial, cuando el gobierno o el contratista, sin hacer una búsqueda de patentes, sepa o tenga motivos demostrables para saber que una patente válida es o será utilizada por o para el gobierno, se informará sin demora al titular de los derechos". Esta norma no es aplicable a licencias obligatorias distintas de aquellas otorgadas para "uso público no comercial". En el caso de la legislación chilena no se prevé una norma que obligue a anticipar al titular de los derechos el eventual uso que se haga de su patente; sin embargo, las normas de procedimiento para el otorgamiento de una licencia obligatoria en cualquier caso garantizan oportuna notificación al titular de la patente.

${ }^{44}$ Artículo 31 a) ADPIC.

${ }^{45}$ Artículo 51 bis C inciso 1 Ley No 19.039.

46 PIRES, The TRIPS, cit. nota n. 9, pp. 318-319, refiere a la prohibición de una "blanket licensing clause".

${ }^{47}$ Correa, Carlos, Intellectual Property Rights and the Use of Compulsory Licenses: Options for Developing Countries, South Center, Ginebra, 1999, pp. 7-8. Correa afirma que tal características es lo que precisamente diferencia a las licencias obligatorias (artículo 31 ADPIC) de las excepciones (artículo 30 ADPIC). En igual sentido, Cabanellas de las Cuevas, Guillermo, Derecho de Patentes de Invención, Tomo II, Heliasta, Buenos Aires, 2004, pp. 422-423.

${ }^{48}$ Artículo 51 bis $C$ inciso 2 Ley No 19.039, en relación con artículo 31 c) ADPIC.

${ }^{49}$ Artículo 51 bis C inciso 2 Ley № 19.039, en relación con artículo 31 d) y e) ADPIC, 5 A) 4) Convención de París.

${ }^{50}$ Artículo 31 h) segunda parte ADPIC. Correa afirma que el artículo 31 k) ADPIC puede ser interpretado en el sentido de permitir "royalty free license", tal como Estados Unidos ha hecho en el pasado. 
suministra criterios de cálculo y ello crea problemas de incertidumbre ${ }^{51}$; la literatura sugiere diversos criterios que pueden ser seguidos, unos más permisivos que otros ${ }^{52}$.

La ley chilena establece que, al concederse una licencia obligatoria, se deberá fijar el monto de la remuneración que se pagará periódicamente por el licenciatario al titular de la patente ${ }^{53}$. Ello hace suponer que la remuneración que el licenciatario pagará al titular de la patente, cualquiera sea la fórmula empleada para su cálculo, se devengará por períodos sucesivos; lo cual guarda consistencia con la extensión temporal de la licencia.

Desafortunadamente, la ley chilena no precisa cómo ni sobre qué base se verificará el cálculo del monto de la remuneración. En tal sentido sería recomendable que, siguiendo la práctica de otros países, se precisaran reglas al respecto, a través de un sistema predecible y fácil de administrar, que garantice transparencia y eficacia ${ }^{54}$.

CorRea, Intellectual, cit. nota n. 47, pp. 7-8. En sentido contrario, Cabanellas, Derecho de patentes, cit. nota n. 47, p. 329. Gómez interpreta el 31 k) como una aminorante, pero nunca como una completa eliminación de la remuneración. Gómez Velasco, Xavier, Patentes de Invención y Derecho de la Competencia Económica, Universidad Andina Simón Bolívar, Quito, 2003, p. 118. La afirmación de Correa es cierta, eventualmente la reducción puede conducir a la completa eliminación de la remuneración; sin embargo, esta hipótesis debe entenderse circunscrita a aquellas licencias obligatorias otorgadas ante prácticas anticompetitivas.

${ }^{51}$ SCHERER, F. M., "The Pharmaceutical Industry and World Intellectual Property Standards", 53 Vanderbilt Law Review 2245, 2000, p. 2252; PIRES, The TRIPS, cit. nota n. 9, p. 366.

${ }^{52}$ Entre los criterios maximalistas, podemos mencionar a Pires de Carvallo, quien asegura que tratándose de licencias compulsorias la remuneración debe ser calculada "exactamente en el monto de la ganancia financiera real y potencial que la licencia obligatoria puede extraer del mercado... (y también) el costo de elaboración de los datos... debe verse reflejado en la compensación adecuada que debe ser pagada al titular de la patente". PIRES, The TRIPS, cit. nota n. 9, pp. 366-367. Por su parte, una aproximación minimalista tiene el Banco Mundial, que en casos de salud limita a "la más baja regalía basada en el precio de compra de las medicinas genéricas", citado por Аввотт; CotTIER; Gurry, International, cit. nota. n. 34, pp. 197-198. Correa, siguiendo el ejemplo de Canadá e India, sugiere "un porcentaje de las ventas netas, tomando en consideración el valor de la licencia en el mercado interno relevante y las regalías promedio usualmente pagadas en el sector o rama a que la invención pertenece". CORREA, Carlos, Integrating Public Health Concerns into Patent Legislation in Developing Countries, South Center, Ginebra, 2000, pp. 106-108. En similar sentido, Love sugiere fijar la remuneración en una proporción de los royalties asociado al nivel de innovación de los productos. Love, James, "Access to Medicine and Compliance with the WTO TRIPS Accord: Models for State Practices in Developing Countries", en Drahos, Peter; MaYne, Ruth (Edits.), Global Intellectual Property Rights. Knowledge, Access and Development, Palgrave Macmillan, Nueva York, 2002, pp. 78, 84-86.

${ }^{53}$ Artículo 51 bis C inciso 2 Ley № 19.039.

${ }^{54}$ Love, "Access", cit. nota n. 52, pp. 78, 83-84. Love sugiere que, tratándose de productos altamente sofisticados que requieren múltiples patentes, se prevea un fondo común ("escrow funds") que financie a los titulares de patentes implicadas. 
Revisión judicial. El Acuerdo sobre los ADPIC requiere que tanto la decisión relativa al otorgamiento de una licencia obligatoria, como la relativa a la remuneración prevista en ella, sean susceptibles de revisión judicial u otra revisión independiente por una autoridad superior diferente del mismo país ${ }^{55}$. En el caso de Chile, las decisiones del Director Nacional del INAPI sobre licencias obligatorias fundada en razones de salud pública pueden ser revisadas judicialmente por el Tribunal de Propiedad Industrial y, eventualmente, por la Corte Suprema ${ }^{56}$.

Revocación de la licencia. La revocación de la licencia es también consistente con el Acuerdo sobre los ADPIC ${ }^{57}$. El INAPI puede dejar sin efecto la licencia obligatoria otorgada, total o parcialmente, a reserva de los intereses legítimos del licenciatario, en caso de que las circunstancias que la justificaban hayan desaparecido y no sea probable que vuelvan a surgir ${ }^{58}$. La revocación no tendrá lugar si fuese probable que se repitieran las circunstancias que dieron origen a su concesión ${ }^{59}$.

La legislación chilena en materia de patentes obligatorias fundadas en razones de salud pública no se limitó a cumplir literalmente con el Acuerdo sobre los ADPIC. Es posible identificar cuando menos cuatro puntos en los cuales difiere del tenor de los Acuerdos, a saber: la posibilidad de disponer de licencias obligatorias provisionales, la modificación de las mismas, la garantía de acceso a datos de prueba y el rechazo a limitar las licencias a "abastecer el mercado interno". Revisemos las primeras tres de tales innovaciones, mientras que la última de ellas la analizaremos conjuntamente con la Declaración de Doha y la subsecuente Decisión.

Licencia Provisional. Mediante resolución fundada, el Director Nacional del INAPI puede acceder provisionalmente a la solicitud de licencia obligatoria ${ }^{60}$.

\footnotetext{
${ }^{55}$ Artículo 31 i) y j) ADPIC.

${ }^{56}$ Artículos 51 bis B 2), 18 bis O, 17, y 17 bis B Ley № 19.039 .

${ }^{57}$ Artículo 31 g) y k) ADPIC.

${ }^{58}$ Artículo 51 bis D inciso 1 Ley No 19.039 .

${ }^{59}$ Artículo 51 bis D inciso 2 Ley No 19.039 .

${ }^{60}$ Durante la discusión legislativa se decidió eliminar la exigencia de "casos graves y urgentes" para el otorgamiento de una licencia provisional. Ésta podía ser interpretada como una exigencia adicional, en circunstancias que el acceso provisional a la licencia obligatoria ya estaba circunscrito a casos fundados en razones de salud pública, seguridad nacional, uso público no comercial, o de emergencia nacional u otras de extrema urgencia. Nuevo Segundo Informe de la Comisión de Economía (del Senado) recaído en el proyecto de ley que modifica la Ley 19.039, que establece normas aplicables a los privilegios industriales y protección de los derechos de propiedad industrial, 17 de Junio de 2004, p. 10. En: http://sil.congreso.cl/cgi-bin/sil_abredocumentos.pl?2,6559 [visitado el 21/07/2010]. Diario

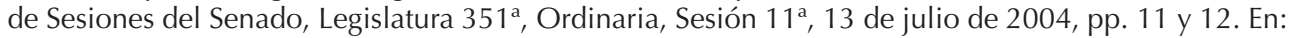
http://sil.congreso.cl/cgi-bin/sil_abredocumentos.pl?5,4668 [visitado el 21/07/2010].
} 
Dicha decisión se mantendrá en vigor mientras duren los fundamentos de hecho que la motivaron o hasta la sentencia definitiva ${ }^{61}$. Aunque la medida no se contempla expresamente en el Acuerdo sobre los ADPIC, ella está ciertamente en armonía con los objetivos del Acuerdo, en orden a permitir una reacción eficaz ante casos de emergencia ${ }^{62}$ : a fast track procedure ${ }^{63}$.

Modificación de la licencia. El INAPI está facultado para modificar la licencia cuando nuevos hechos o circunstancias lo justifiquen ${ }^{64}$. Aunque la modificación no está prevista en el Acuerdo sobre los ADPIC, ella guarda armonía con su intención de que la licencia se limite al cumplimiento de los fines para los que ha sido otorgada ${ }^{65}$. Además, ello permite que las partes continúen en tratativas para el otorgamiento de una licencia voluntaria, aun una vez otorgada la licencia obligatoria.

Acceso a datos de prueba. La legislación de Chile garantiza reserva de los datos de prueba u otros que tengan naturaleza de no divulgados, relativos a la seguridad y eficacia de un producto farmacéutico ${ }^{66}$. Dicha protección no procede, entre otras circunstancias, cuando el producto farmacéutico es objeto de una licencia obligatoria ${ }^{67}$. De este modo, el legislador allana el esfuerzo del licenciatario para producir y comercializar los productos, al permitirle acceder a información que bajo otras circunstancias sería reservada ${ }^{68}$.

\section{La Declaración de Doha y la decisión sobre su aplicación}

Durante la discusión legislativa de la ley que modificó la normativa sobre patentes, algunos parlamentarios expresaron su preocupación por el impacto que la ley pudiese tener en relación a la adquisición de medicamentos genéricos

\footnotetext{
${ }^{61}$ Artículo 51 bis B 2) Ley No 19.039.

${ }^{62}$ El acceso provisional a la demanda de una licencia obligatoria sólo está permitido al Director Nacional del Instituto Nacional de la Propiedad Intelectual, en el caso de solicitudes fundadas por razones de salud pública.

${ }^{63}$ La posibilidad de implementar procedimientos de salida rápida en el derecho interno de los Miembros ha sido sugerida, sobre la base de distintos términos, por diversos autores, entre ellos: Love, "Access", cit. nota n. 52, p. 79; MAYNE, Ruth, "The Global Campaign on Patents and Access to Medicines: An Oxfam Perspective", en Drahos; Mayne, Global, cit. nota n. 52, p. 254.

${ }^{64}$ Artículo 51 bis D inciso 2 Ley No 19.039.

${ }^{65}$ Artículo 31 c) ADPIC.

${ }^{66}$ Artículo 89 Ley № 19.039.

${ }^{67}$ Artículo 91 c) Ley No 19.039.

${ }^{68}$ La imposibilidad de acceder a los datos de prueba puede obstaculizar el otorgamiento y la eficacia de una licencia obligatoria respecto de productos farmacéuticos. Vid., CorreA, Integrating, cit. nota n. 52, pp. 102-104.
} 
sin royalties provenientes de Brasil e India ${ }^{69}$. Al respecto el Gobierno manifestó que el proyecto intentaba "fortalecer los derechos de propiedad industrial... con la precaución de asegurar a los consumidores el acceso a los frutos de la innovación"..$^{70}$ Para lograr tal balance, la ley contaba con ciertas exclusiones de patentabilidad y, además, el proyecto preveía las licencias obligatorias y el agotamiento internacional de los derechos ${ }^{71}$.

El agotamiento internacional permite efectuar importaciones paralelas, esto es, la adquisición de medicamentes en otros países. Ello faculta a un país para afrontar una emergencia de salud comprando los medicamentos necesarios en el extranjero, de ser necesario. Sin embargo, el agotamiento de los derechos no autoriza a conceder una licencia obligatoria para la importación u exportación de tales medicamentos. ¿Cómo lograr el abastecimiento del mercado mediante una licencia obligatoria cuando el país carece de capacidad para producir los medicamentos? ¿Pueden importarse y/o exportarse medicamentos elaborados bajo licencia obligatoria de un país a otro? Responder a estas preguntas nos lleva a la Declaración de Doha y a la Decisión sobre su aplicación.

A finales de la década de los 90 existía incertidumbre entre los países menos desarrollados en cuanto a cómo armonizar la protección de la propiedad intelectual sobre los productos farmacéuticos y sostener sus programas de salud pública, particularmente, en orden a garantizar acceso a las medicinas a precios razonables. La ambigüedad del Acuerdo sobre los ADPIC, el temor a represalias de otros miembros de la OMC y la presión local de las compañías farmacéuti$\operatorname{cas}^{72}$, requerían una urgente implementación e interpretación de los Acuerdos que apoyase "la salud pública, promoviendo tanto el acceso a los medicamentos

\footnotetext{
${ }^{69}$ Diario de Sesiones del Senado, Legislatura 348 , Extraordinaria, Sesión $1^{\text {a }}, 1^{\circ}$ de octubre de 2002, pp. 46-47.

La posibilidad de adquirir medicamentes genéricos en terceros países, especialmente aquellos con políticas más laxas en cuanto a la protección de la propiedad intelectual, era vista como una fórmula de salida para casos de urgencia. Sin embargo, la progresiva adopción del Acuerdo sobre los ADPIC impide acceder a copias genéricas de productos patentados. De ahí la necesidad de encontrar una solución para garantizar acceso a medicamentos especialmente en países en desarrollo. Vid., CommisSION On INTELleCtual Property Rights, Integrating Intellectual Property Rights and Development Policy, Londres, 2002, pp. 41-42. Por ejemplo, durante los años 50, eludiendo los altos costos de Pfizer, Estados Unidos hizo adquisición de genéricos de tetraciclina en Italia, país que para entonces no protegía los fármacos con patentes. Vid., SCHerer, "The Pharmaceutical", cit. nota. n. 51, p. 2250.

${ }^{70}$ INFORME DE HACIENDA (del Senado) recaído en el proyecto de Ley de la H. Cámara de Diputados Sobre Propiedad Industrial, 14 de mayo de 2004, p. 3. En: http://sil.congreso.cl/cgi-bin/sil_abredocumentos. pl?2,6560 [visitado el 21/07/2010].

${ }^{71}$ INFORME DE HACIENDA, cit. nota n. 70. El agotamiento internacional de los derechos se contempla en el artículo 49 inciso 5 de la Ley № 19.039 .

${ }^{72}$ Estos hechos son detalladamente descrito en SelL, Private, cit. nota. n. 3, pp. 146-162.
} 
existentes como la investigación y desarrollo de nuevos medicamentos ${ }^{173}$; ello se verificó a través de la Declaración de Doha ${ }^{74}$.

Con el afán de resolver los problemas suscitados en torno al Acuerdo de los ADPIC, la Declaración de Doha clarificó las flexibilidades existentes ${ }^{75}$; la Declaración no estableció nuevas reglas, sino que interpretó aquellas ya disponibles en el Acuerdo ${ }^{76}$. En breve, dicho Acuerdo no impide ni deberá impedir que los miembros adopten medidas para proteger la salud pública, y reafirma el derecho de los miembros de la OMC de utilizar, al máximo, las disposiciones del Acuerdo sobre los ADPIC, que prevén flexibilidad a este efecto ${ }^{77}$. Los países miembros tienen, entre otros: el derecho de conceder licencias obligatorias, el derecho de determinar lo que constituye una emergencia nacional u otras circunstancias de extrema urgencia, y la libertad de establecer su propio régimen de agotamiento de los derechos de propiedad intelectual ${ }^{78}$.

Sin embargo, la Declaración de Doha dejó un asunto clave sin resolver ${ }^{79}$. De acuerdo al artículo $31 \mathrm{f}$ ) del Acuerdo sobre los ADPIC, las licencias obligatorias se autorizarían "principalmente" para abastecer el mercado interno ${ }^{80}$; en otras palabras, ningún país podría otorgar una licencia obligatoria para abastecer principalmente o exclusivamente otro mercado. Esta exigencia se había transformado en un serio inconveniente, especialmente para aquellos países con insuficiencia o carencia de capacidad de manufactura en el sector farmacéutico. Éste constituía un verdadero problema de doble entrada: desde el lado de la demanda, porque los países menos desarrollados carecían de capacidades; $y$, desde el lado de la oferta, por la limitación a proveer "principalmente" al mercado interno prevista en el Acuerdo ${ }^{81}$.

¿Cómo podría un país con insuficiencia o carencia de capacidad de manufactura hacer un uso efectivo del mecanismo de licencias obligatorias

\footnotetext{
${ }^{73}$ Párrafo 17 Declaración Ministerial de Doha, adoptada el 14 de noviembre de 2001, por la Cuarta Conferencia Ministerial de la OMC, Doha, Qatar. WT/MIN(01)/DEC/1. 20 de noviembre de 2001.

${ }^{74}$ Declaración relativa al Acuerdo sobre los ADPIC y la salud pública, adoptada el 14 de noviembre de 2001, por la Cuarta Conferencia Ministerial de la OMC, Doha, Qatar. WT/MIN(01)/DEC/2. 20 de noviembre de 2001.

${ }^{75}$ MAYNe, "The Global", cit. nota n. 63, p. 253.

${ }^{76}$ Аввотт; СотtIer; GurRY, International, cit. nota. n. 34, p. 200.

${ }_{77}$ Párrafo 4 Declaración relativa al Acuerdo sobre los ADPIC y la salud pública, supra.

${ }^{78}$ Párrafo 5 Declaración relativa al Acuerdo sobre los ADPIC y la salud pública, supra.

${ }^{79}$ Párrafo 6 Declaración relativa al Acuerdo sobre los ADPIC y la salud pública, supra.

${ }^{80} \mathrm{El}$ artículo $31 \mathrm{f}$ ) de Acuerdo sobre los ADPIC ha sido analizado extensamente en la literatura. Vid., CORREA, Carlos, Implications of the Doha Declaration on the Trips Agreement and Public Health, Health Economics and Drugs Series No 012, Organización Mundial de la Salud, 2002; y, PIRES, The TRIPS, cit. nota n. 9, pp. 329-365.
}

${ }^{81}$ Аввотт; CotтIer; Gurry, International, cit. nota. n. 34, pp. 205-206. 
adoptado en el Acuerdo sobre los ADPIC? La respuesta para tal interrogante debió todavía esperar hasta la adopción de una nueva Decisión por el Consejo General de la $\mathrm{OMC}^{82}$. Eximiendo a los países de dar cumplimiento a la exigencia del $31 \mathrm{f}$ ) del Acuerdo sobre los ADPIC, la Decisión creó un sistema que permite la exportación de productos farmacéuticos desde un país a otro, si ambos países satisfacían los requisitos y procedimientos establecidos en la Decisión.

El mecanismo adoptado por la decisión podría ser empleado para proveer cualquier producto farmacéutico, para cualquier enfermedad y por cualquier país ${ }^{83}$. El sistema podría ser empleado por un país menos desarrollado, pero también por cualquier otro país una vez éste ha notificado al Consejo de los ADPIC su intención de emplear el sistema como importador ${ }^{84}$. No obstante, algunos países declararon que no harían uso del mecanismo como importadores, en tanto que otros declararon que, en caso de usar el sistema, lo harían sólo en limitadas situaciones. Además, la Decisión incluye varios medidas de resguardo para evitar la desviación de los productos del mercado, por ejemplo, mediante la comercialización y empaquetado de aquéllos con características identificables ${ }^{85}$.

El sistema creado por la Decisión era una solución transitoria ${ }^{86}$, pero éste devino en el mecanismo final una vez que los miembros de la OMC aceptaron

\footnotetext{
${ }^{82}$ Decisión del Consejo General de 30 de agosto de 2003 sobre Aplicación del párrafo 6 de la Declaración de Doha relativa al Acuerdo sobre los ADPIC y la salud pública. WT/L/540 and Corr.1. $1^{\circ}$ de septiembre de 2003.

${ }^{83}$ Una propuesta para limitar el sistema a una lista de medicinas, enfermedades o países fue rechazada. Vid., Paragraph 6 of the Doha Declaration on the TRIPS Agreement and Public Health - Communication from the United States, WTO document IP/C/W/340, March 14th, 2002; FERGusson, lan, The WTO, Intellectual Property Rights, and the Access to Medicines Controversy, Congressional Research Service - The Library of Congress, Washington, November 5, 2003; y, SEK, Lenore, World Trade Organization Negotiations: The Doha Development Agenda, Congressional Research Service - The Library of Congress, Washington, August 6, 2004.

${ }^{84} \mathrm{El}$ sistema es consistente con la idea de que "la insuficiencia o ausencia de capacidad de manufactura" es un concepto relativo, ya que inclusive un país desarrollado podría ser incapaz de producir una determinada medicina en determinadas circunstancias. Vid., CORREA, Implications, cit. nota n. 80, p. 23. Un país menos desarrollado se presume carece de dicha capacidad, mientras otros países deben notificar y confirmar la insuficiencia o carencia de capacidad de manufactura. Este es un proceso de autodeterminación, sin revisión formal, a diferencia de lo que fuera propuesto por los Estados Unidos. FerGuSSON, The WTO, cit. nota n. 83, p. 5. De hecho, de acuerdo a la nota al pie número 8 de la Decisión, la notificación no necesita ser aprobada por ningún órgano de la OMC.

${ }^{85}$ Una propuesta destinada a limitar el sistema a programas humanitarios de salud pública, a cargo de gobiernos o de organizaciones de caridad, fue también rechazada. FERGUSSON, The WTO, cit. nota n. 83, p. 5.

${ }^{86}$ Párrafo 11 Decisión del Consejo General de 30 de agosto de 2003, sobre Aplicación del párrafo 6 de la Declaración de Doha relativa al Acuerdo sobre los ADPIC y la salud pública. WT/L/540 y Corr.1
} 
la Enmienda adoptada por el Consejo General de la $\mathrm{OMC}^{87}$. El Protocolo de Enmienda introduce una modificación permanente al texto del Acuerdo sobre los ADPIC que intenta resolver el inconveniente de aquellos países con insuficiencia o carencia de capacidad de manufactura para hacer un uso efectivo del mecanismo de licencias obligatorias. Dicha modificación, en lo sustancial, sigue los términos de la Decisión precedente.

El Protocolo de Enmienda está aún abierto para su aceptación ${ }^{88}$, y entrará en vigencia una vez que dos tercios de los miembros de la OMC lo hayan aceptado. Actualmente, 56 países han aceptado la Enmienda ${ }^{89}$. Por su parte, aun cuando Chile expresó su satisfacción por la adopción de la Decisión en su día, todavía no ha aceptado el Protocolo ${ }^{90}$.

\section{V. ¿Debería Chile aceptar el Protocolo de Enmienda?}

Para responder a la pregunta que antecede es necesario, a lo menos, evaluar brevemente el sistema de licencias obligatorias, y hacer una sucinta referencia al consumo y producción de productos farmacéuticos en Chile.

Evaluar el sistema adoptado por la OMC para licencias obligatorias puede ser precipitado, especialmente al analizar sólo el sistema establecido por la Decisión y el Protocolo de Enmienda. De hecho, no existe acuerdo entre los expertos sobre el particular: algunos de ellos estiman que las licencias son peligrosas para los países y los consumidores, y preferirían limitar sus efectos

\footnotetext{
${ }^{87}$ Decisión sobre la Enmienda del Acuerdo sobre los ADPIC, adoptada por el Consejo General, el 6 de diciembre de 2005. WT/L/64. 8 de diciembre de 2005. Hay mínimas diferencias entre la Decisión y la Enmienda, en general destinadas a clarificar ciertas disposiciones que evitan doble pago y que regulan las economías de escala en acuerdos comerciales regionales. No estaba claro si la decisión permitía una exención a la remuneración en el país exportador o sólo "un procedimiento para evitar el pago doble". PIRES, The TRIPS, cit. nota n. 9, p. 367.

${ }^{88} \mathrm{El}$ Protocolo fue inicialmente abierto para su aceptación por los miembros de la OMC hasta el $1^{\circ} \mathrm{de}$ diciembre de 2007, pero el plazo fue extendido hasta el 31 de diciembre de 2009 o la fecha posterior que pueda ser decidida por la Conferencia Ministerial. Vid., Decisión sobre Enmienda del Acuerdo sobre los ADPIC - Prórroga del Plazo para la Aceptación por los Miembros del Protocolo por el que se Enmienda el Acuerdo sobre los ADPIC, adoptado por el Consejo General, el 18 de diciembre de 2007. WT/L/711. 21 de diciembre de 2007. Posteriormente, dicho plazo fue extendido nuevamente hasta el 31 de diciembre de 2011 o una fecha posterior que pueda decidir la Conferencia Ministerial. Vid., Decisión sobre Enmienda del Acuerdo sobre los ADPIC - Segunda Prórroga del Plazo para la Aceptación por los Miembros del Protocolo por el que se Enmienda el Acuerdo sobre los ADPIC, adoptado por el Consejo General, el 17 de diciembre de 2009. WT/L/785. 21 de diciembre de 2009.

${ }^{89}$ La lista de estos países está En: http://www.wto.org/english/tratop_e/trips_e/amendment_e.htm. 29 países y la Unión Europea, integrada por otros 27 países, han aceptado la Enmienda, al 15 de julio de 2010 [visitado el 21/07/2010].

${ }^{90}$ Minutes of Meeting General Council on 25, 26 and 30 August 2003. WTO document WT/GC/M/82. 13 November 2003.
} 
mediante el incremento de los requisitos para su otorgamiento ${ }^{91}$; otros autores sugieren que inclusive el actual mecanismo previsto en la Enmienda no debería ser adoptado, porque es insuficiente, burocrático e inapropiado para hacer frente a su socavación por acuerdos comerciales bilaterales y regionales ${ }^{92}$. Al mismo tiempo, no existe acuerdo sobre la eficacia del mecanismo previsto en la Decisión y el Protocolo de Enmienda entre los países miembros de la OMC, evidenciando una clara distinción en la evaluación que hacen los países desarrollados de aquellos en desarrollo ${ }^{93}$.

Sin embargo, la mayor parte de los autores está de acuerdo en que, inclusive cuando las licencias obligatorias han sido usadas de modo muy limita$\mathrm{do}^{94}$, ellas reducen el costo de acceso a medicamentos para la gente, ya sea por la sola amenaza que implican para los titulares de las patentes y/o por su efectivo otorgamiento ${ }^{95}$. Por ejemplo, en 2001, una amenaza de licencia obligatoria permitió al gobierno de Brasil asegurar el acceso universal a su programa HIV/AIDS, al lograr una significativa reducción en los precios de

\footnotetext{
${ }^{91}$ BIRD, Robert, "Developing Nations and the Compulsory License: Maximizing Access to Essential Medicines While Minimizing Investment Side Effects", 37 Journal of Law Medicine \& Ethics, 209. (Summer 2009). Bird sostiene que las licencias obligatorias no deberían ser excesivamente amplias, deberían ser redactadas en consulta con los titulares de patentes, y limitadas a genuinas metas humanitarias y solamente para medicinas que permiten salvar vidas.

${ }^{92}$ Bradford, Vanessa; LeE, Kalley, "TRIPS, the Doha Declaration and Paragraph 6 Decision: What Are the Remaining Steps for Protection Access to Medicines?", Globalization and Health, 2007, 3:3. Para una referencia acerca de las limitaciones que los acuerdos de libre comercio introducen en relación con el acceso a las medicinas y la regulación de la propiedad intelectual sobre las mismas, vid., Roffe, Pedro; SANTA CRUZ, Maximiliano, Los derechos de propiedad intelectual en los acuerdos de libre comercio celebrados por países de América Latina con países desarrollados, Serie Comercio Internacional, Comisión Económica para América Latina y el Caribe, CEPAL, 2006, pp. 49-53.

${ }_{93}$ Vid., Los Miembros preguntan: ¿Está funcionando el sistema del párrafo 6 sobre propiedad intelectual y salud?, Noticias OMC, 2 de marzo de 2010. En: http://www.wto.org/spanish/news_s/news10_s/ trip_02mar10_s.htm\#12feb_consult [visitado el 21/07/2010].

${ }^{94}$ A pesar de las flexibilidades provistas por el Acuerdo sobre los ADPIC, hay un reducido número de casos en que los países han adoptado licencias obligatorias por razones de salud pública u otras similares. De hecho, el sistema adoptado por la Decisión ha sido usado sólo una vez, por Rwanda como exportador y Canadá como importador. Vid., Notification under paragraph 2(a) of the Decision of 30 August 2003 on the Implementation of paragraph 6 of the Doha Declaration on the TRIPS Agreement and Public Health - Rwanda, WTO document, P/N/9/RWA/1. 19 July 2007; y, Notification under Paragraph 2(c) of the Decision of 30 August 2003 on the Implementation of paragraph 6 of the Doha Declaration on the TRIPS Agreement and Public Health - Canada, WTO document, IP/N/10/CAN/1. 8 October 2007.

${ }^{95}$ Correa, Intellectual, cit. nota n. 47, pp. 22-23; Fergusson, The WTO, cit. nota. 83, p. 6; АввотT; CotTIer; GuRRY, International, cit. nota. n. 34, pp. 205-206; CARVAJAL, Yuri, "Patentes Farmacéuticas y Acuerdos Comerciales", Cuadernos Médico Sociales (Chile) 49 (2), 2009, pp. 111-122.
} 
los medicamentos antiretrovirales (entre un 40 y $64.8 \%$ de reducción en sus (costos) ${ }^{96}$.

En Chile existe un alto consumo de medicamentos, preferiblemente genéricos a un menor precio. De hecho, de acuerdo al Servicio Nacional del Consumidor, Chile es el tercer país en consumo y ventas de medicamentos per capita en Latinoamérica97. De tal consumo, durante el 2009, de acuerdo a cifras de la asociación de farmacéuticas local, un 19,4\% de los medicamentos vendidos eran de marca contra un $80,6 \%$ de genéricos; sin embargo, las marcas representaron un $41,3 \%$ del valor del mercado contra sólo un $58,7 \%$ de los genéricos; de hecho, el valor promedio de los medicamentos es: 10,0 dólares para las marcas, 5,3 dólares para los similares, y sólo 0,8 dólares para los genéricos ${ }^{98}$.

Chile tiene una relativa capacidad productiva y exportadora de productos farmacéuticos. Respecto de la oferta de medicamentos en Chile, en 2009 un $80,4 \%$ de las ventas correspondió a medicamentos producidos por farmacéuticas locales ${ }^{99}$. Además, ese mismo año, aun cuando las importaciones excedieron a las exportaciones, Chile exportó más de 105 millones de dólares en productos farmacéuticos ${ }^{100}$.

Considerando que el Acuerdo sobre los ADPIC provee flexibilidades que los países miembros deberían emplear, especialmente aquellas relativas a licen-

${ }^{96}$ Oliveira, María Auxiliadora et al., "Has the Implementation of the TRIPS Agreement in Latin America and the Caribbean Produced Intellectual Property Legislation that Favors Public Health?", en Bulletin of the World Health Organization, 2004; 82:815-821.

${ }^{97}$ Servicio Nacional del Consumidor, Grandes Cadenas de Farmacias: Qué Hay Detrás de las Promesas Publicitarias, junio 2004 (Anexos), p. 13.

${ }^{98}$ Asociación Industrial de Laboratorios Farmacéuticos (ASILFA), Estadísticas 2009. En: http://www.asila. cl [visitado el 15/07/2010]. Según informaciones de prensa, sobre la base de los 10 medicamentos más comprados por los chilenos, existen diferencias de hasta $2.682,6 \%$ entre los remedios de marca y los genéricos. Así, el analgésico y antiinflamatorio Lertus vale 12 dólares, mientras el genérico diclofenaco sódico, en iguales dosis y comprimidos, menos de 0,50 dólares; mientras, el anticolesterol Lipitor vale aproximadamente 40 dólares y su equivalente menos de 2 dólares. La Tercera, Diferencias de hasta $2.600 \%$ muestran precios de un mismo remedio (Valeria Ibarra), 10 mayo 2009, pp. 46-47.

Resultados similares han sido encontrados en otros países. En Brasil, por ejemplo, un estudio desarrollado entre 2000 y 2004 demostró que los medicamentos genéricos son en promedio un 40\% más baratos que los de marca. Sulpino VieIRA, Fabiola; ZucchI, Paola, "Diferenças de preços entre medicamentos genéricos e de referência no Brasil: Price differences between generic and innovator medicines in Brazil", Revista de Saúde Pública 40 (3), 2006, pp. 444-449. En los Estados Unidos, los medicamentos reducen sus costos más de un $85 \%$ dentro del año siguiente a la expiración de la patente. The Economist, Generically Challenged: The Pharmaceucals Industry May Yet Find Inspiration in Its Old Nemesis, the Generic-Drug Business (Vijay Vaitheeswaran), November 13th, 2009.

${ }^{99}$ Según ASILFA, las exportaciones ascendieron a 105.488.064,03 dólares, mientras las importaciones llegaron a 627.286.664,11 dólares. Asociación Industrial de Laboratorios Farmacéuticos (ASILFA), Estadísticas 2009. En: http://www.asilfa.cl [visitado el 21/07/2010].

100 Ídem. 
cias obligatorias, que las cifras muestran una relativa capacidad productiva y exportadora de las empresas farmacéuticas locales que es deseable preservar y estimular ${ }^{101}$, y que es esencial para la salud pública del país preservar y disponer de medidas que resguarden ese acceso de la población a medicinas, parece completamente recomendable aceptar el Protocolo de Enmienda al Acuerdo sobre los ADPIC e implementar las flexibilidades provistas por éste en el derecho interno de nuestro país.

En el caso de Chile, la implementación de la Decisión desde la perspectiva de la importación requiere un simple decreto administrativo, mientras que la implementación de la misma desde la perspectiva de la exportación requeriría una modificación legal ${ }^{102}$. Afortunadamente, Chile podría capitalizar sobre la base de la experiencia de otros países en la materia. Canadá, Corea del Sur, China, India y Noruega han adoptado modificaciones legales con exenciones que permiten a las industrias farmacéuticas de genéricos manufacturar y exportar medicinas bajo una licencia obligatoria de acuerdo al sistema previsto en la $\mathrm{OMC}^{103}$. La Unión Europea hizo igual ${ }^{104}$, y en los Estados Unidos se ha introducido un proyecto de ley que establece los procedimientos para el otorgamiento de tal tipo de licencias obligatorias ${ }^{105}$.

\section{CONCLUSIONES}

Al adoptar la Ley № 19.996 en 2005, a pesar de eludir la discusión concerniente al artículo 31 f) del Acuerdo sobre los ADPIC, Chile cumplió con las disposiciones del Acuerdo en materia de licencias obligatorias y ha implementado las flexibilidades existentes respecto del otorgamiento de tales licencias

101 Es interesante constatar el potencial que las empresas farmacéuticas locales, tanto por sus costos de producción como por su acceso al mercado interno, en especial para la población local de mejores ingresos. Estas características han movido a diversas farmacéuticas internacionales a estrechar lazos de colaboración con las empresas locales, porque "los genéricos ya no son los enemigos de la innovación farmacéutica". Vid., The Economist, "Big Drug Firms Embrace Generics: Friend for Life", August 6, 2009.

102 De acuerdo a Correa, en cualquier caso, la implementación de la solución desde la perspectiva de la parte exportadora requiere una modificación en el derecho interno. CORREA, Implications, cit. nota. n. 47, pp. 24, 25, 36 y 49. En el caso de Chile, la modificación legal es también necesaria por exigencias constitucionales. Vid., artículo $19 \mathrm{~N}^{\circ} 24$ y No 25 de la Constitución.

${ }^{103}$ Fergusson, Ian, The WTO, Intellectual Property Rights, and the Access to Medicines Controversy, Congressional Research Service - The Library of Congress, Washington, November 5, 2007, p. 8.

${ }^{104}$ Regulation (EC) No 816/2006 of the European Parliament and of the Council of 17 May 2006 on compulsory licensing of patents relating to the manufacture of pharmaceutical products for export to countries with public health problems, Official Journal of the European Union, 09.06.2006.

${ }^{105}$ La Life-Savings Medicines Export Act de 2006. Una breve descripción de la propuesta de ley en FergusSON, The WTO, cit. nota. n. 103, p. 6. 
por razones de salud pública. La ley chilena vigente es, además, consistente con la Declaración de Doha. Sin embargo, nuestra ley no saca ventaja de las flexibilidades provistas posteriormente en la Decisión ni en el Protocolo de Enmienda ${ }^{106}$. La actual normativa nacional parece aceptar la importación, a pesar de no regular expresamente el sistema adoptado en la mencionada Decisión; en cambio, la ley no permitiría las licencias obligatorias para exportación de productos farmacéuticos.

En consecuencia, y sin perjuicio de cuanto se ha dicho precedentemente, para utilizar, al máximo, las flexibilidades provistas por el Acuerdo sobre los ADPIC, Chile debería aceptar el Protocolo de Enmienda al Acuerdo e implementar dichas flexibilidades en su derecho interno: mediante un simple decreto administrativo, en el caso de la licencia obligatoria para importación; mediante una modificación legal, en el caso de la licencia obligatoria para exportación.

\section{BibLIOGRAFÍA}

Аввотт, Frederick; COTTIER, Thomas; GuRRY, Francis, International Intellectual Property in an Integrated World Economy, ASPEN, Nueva York, 2007.

BIRD, Robert, "Developing Nations and the Compulsory License: Maximizing Access to Essential Medicines While Minimizing Investment Side Effects", 37 Journal of Law Medicine \& Ethics, 209. (Summer 2009).

Bradford, Vanessa y LeE, Kalley, "TRIPS, the Doha Declaration and Paragraph 6

Decision: What Are the Remaining Steps for Protection Access to Medicines?", en Globalization and Health, 2007,

BRENNAN, David J., Retransmission and US Compliance with TRIPS, Kluwer Law International, La Haya, Holanda, 2003.

Cabanellas de las Cuevas, Guillermo, Derecho de Patentes de Invención, Tomo II, Heliasta, Buenos Aires, 2004.

Carvajal, Yuri, "Patentes Farmacéuticas y Acuerdos Comerciales", Cuadernos Médico Sociales 49 (2) (Chile) 2009.

COMmission On INTELLECTUAL Property Rights, Integrating Intellectual Property Rights and Development Policy, Londres, 2002.

CORREA, Carlos, Intellectual Property Rights and the Use of Compulsory Licenses: Options for Developing Countries, South Center, Ginebra, 1999. Integrating Public Health Concerns into Patent Legislation in Developing Countries, South Center, Ginebra, 2000.

106 Aun cuando la Ley № 19.996 fue publicada en 2005, ella no toma ventajas de la Decisión (2003) ni de la Enmienda (2005), porque dichos instrumentos fueron adoptados una vez que el debate de dicha ley se encontraba cerrado. 
Implications of the Doha Declaration on the Trips Agreement and Public Health, Health Economics and Drugs Series № 012, Organización Mundial de la Salud, 2002.

, "Pro-competitive Measures under TRIPS to Promote Technology Difussion in Developing Countries", en Drahos, Peter; MaYNe, Ruth (Edits.), Global Intellectual Property Rights. Knowledge, Access and Development, Palgrave Macmillan, Nueva York, 2002.

, "The TRIPS Agreement and Developing Countries", en MACRONY y otros (Edits.), The World Trade Organization: Legal, Economic and Political Analysis, Volumen 2, Springer, New York, 2005.

Fergusson, lan, The WTO, Intellectual Property Rights, and the Access to Medicines Controversy, Congressional Research Service - The Library of Congress, Washington, November 5, 2003.

The WTO, Intellectual Property Rights, and the Access to Medicines Controversy, Congressional Research Service - The Library of Congress, Washington, November 5, 2007.

Gervals, Daniel, The TRIPS Agreement: Drafting, History and Analysis, Thomson Reuters, 3ª Edición, Londres, 2008.

Gómez Velasco, Xavier, Patentes de Invención y Derecho de la Competencia Económica, Universidad Andina Simón Bolívar, Quito, 2003.

HASSON, Adam Isaac, "Domestic Implementation of International Obligations: the Quest for World Patent Law Harmonization", 25 Boston College International and Comparative Law Review 373, 2002.

LOVE, James, "Access to Medicine and Compliance with the WTOTRIPS Accord: Models for State Practices in Developing Countries", en DraHos, Peter; MAYNE, Ruth (Edits.), Global Intellectual Property Rights. Knowledge, Access and Development, Palgrave Macmillan, Nueva York, 2002.

MAYNE, Ruth, "The Global Campaign on Patents and Access to Medicines: An Oxfam Perspective", en Drahos, Peter; Mayne, Ruth (Edits.), Global, Global Intellectual Property Rights. Knowledge, Access and Development, Palgrave Macmillan, Nueva York, 2002.

MONCAYO VON HASE, Andrés, "The Application and Interpretation of the Agreement on Trade-Related Aspect of Intellectual Property Rights", en CorreA, Carlos; Yusuf, Abdulqawi (Edits.), Intellectual Property and International Trade: the

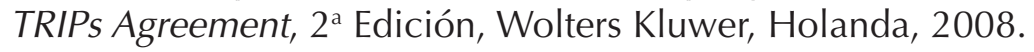

OliveirA, María Auxiliadora y otros, "Has the Implementation of the TRIPS Agreement in Latin America and the Caribbean Produced Intellectual Property Legislation that Favors Public Health?", en Bulletin of the World Health Organization, 2004. 
Pires de Carvallo, Nuno, The TRIPS Regime on Patent Rights, Kluwer Law International, Netherlands, 2005.

Scherer, F. M., "The Pharmaceutical Industry and World Intellectual Property Standards", 53 Vanderbilt Law Review 2245, 2000.

SEK, Lenore, World Trade Organization Negotiations: The Doha Development Agenda, Congressional Research Service - The Library of Congress, Washington, August 6, 2004.

SelL, Susan, Private Power, Public Law: The Globalization of Intellectual Property Rights, Cambridge Studies in International Relations, Cambridge, 2003.

SUlPINO VIEIRA, Fabiola; ZUCCHI, Paola, "Diferenças de preços entre medicamentos genéricos e de referência no Brasil: Price differences between generic and innovator medicines in Brazil", Revista de Saúde Pública, 2006.

RofFe, Pedro; SANTA Cruz, Maximiliano, Los derechos de propiedad intelectual en los acuerdos de libre comercio celebrados por países de América Latina con países desarrollados, Serie Comercio Internacional, Comisión Económica para América Latina y el Caribe, CEPAL, 2006.

UNCTAD-ICTSD, Resource Book on TRIPS and Development, Cambridge University Press, Nueva York, 2005.

WatAL, Jayashree, Intellectual Property Rights in the WTO and Developing Countries, Kluwer Law International, La Haya, Holanda, 2001.

WeGner, Harold C., "Injunctive Relief: A Charming Betsy Boomerang", en Northwestern Journal of Technology and Intellectual Property Volumen 4, Número 2, 2006. 